\title{
Criterios e indicadores para evaluar la calidad del análisis documental de contenido
}

\author{
José Antonio Moreiro \\ Departamento de Biblioteconomía y Documentación \\ Universidad Carlos III de Madrid \\ mailto: jamore@bib.uc3m.es
}

\begin{abstract}
Resumen
Revisión de los criterios que se deben aplicar en una medición de la calidad de los productos surgidos en los procesos del análisis documental de contenido. La calidad de las tareas correspondientes de manera especial a la indización y al resumen recibe su importancia de permitir una satisfactoria recuperación de información y una adecuada explicación de los contenidos a los usuarios. Se describen los rasgos y criterios que marcan un proceso que no puede limitarse a una medición estadística y numérica de los factores cualitativos, y que solo se pueden apreciar mediante la observación y juicio personales centrados en tareas de identificación de los repertorios evaluados, de valoración de la preparación profesional de los analistas, de la existencia de normativas o directrices en el centro, y del tipo de lenguaje utilizado para efectuar la indización. Estos factores cualitativos o criterios se especifican en el grado de reutilización de los resúmenes y de la indización hechos en origen, en cómo se ha efectuado el traslado de la superestructura, en la calidad técnica, y en la profundidad del análisis. Junto a unas unidades de medida o indicadores (como los de Cobertura y solapamiento de los repertorios, Coherencia, Densidad informativa, Exhaustividad, Indicador temporal, Indicador de costes, Indicador del esfuerzo del usuario, e Indicador de errores, Índice de consistencia, Legibilidad, Pertinencia, Profundidad, Tamaño).
\end{abstract}

\section{Palabras-clave}

Evaluación de la calidad; Análiisis documental; Indización; Resumen; Repertorios; Bases de datos; Criterios cualitativos; Criterios quantitativos; Factores; Indicadores.

\section{Criteria and indicators for evaluating the quality of documental content analysis}

\begin{abstract}
Revision of the criteria which must be applied in measuring the quality of the products coming from processes of documental analysis of content. The quality of the tasks corresponding, especially, to indexing and abstract is important for allowing a satisfactory retrieval of information and an appropriate explanation of the contents to the users. The traits and criteria about a process which cannot be limited only to statistical and numerical measure of the qualitative factors are described. They can only be evaluated through the personal observation and judgment underlined in the tasks of identification of the evaluated repertories, appraisal of professional preparation of the analysts, existence of norms or guidelines in the center, and type of language utilized for carrying out the indexing. These qualitative factors or criteria are specified as they reutilize abstracts and indexing done in the source, as they have carried out the transfer from the superstructure, according to a technical quality, and a thorough analysis. Measuring units or indicators are also included (as for instance, Covering and undermining of the repertories; Coherence; Information density; Exhaustiveness; Temporal indicator; Indicator of costs; Indicator of user effort; and Indicator of errors; Index of coherence; Legibility; Relevance; Depth; Size).
\end{abstract}

\section{Keywords}

Quality evaluation; Documental analysis; Indexing; Abstract; Repertories; Databases; Qualitative Criteria; Quantitative criteria; Factors; Indicators.

\section{JUSTIFICACIÓN Y PROPÓSITOS}

La calidad es un asunto de importancia creciente en los servicios de información. De su seguimiento preocupa ante todo cuanto se refiere a la gestión normalizada de la calidad en los sistemas de información, junto a la propia medición de la calidad que presentan los productos informativos ${ }^{1}$. La norma ISO 8402 define la calidad como "la totalidad de rasgos distintivos de un producto o servicio que tienen que ver con su capacidad para satisfacer


respecto al producto debe verse desde una doble perspectiva: la que se refiere a administrar externamente las expectativas de los usuarios respecto a un producto, $y$ la que desde dentro intenta reducir las consecuencias de los fallos humanos y empequeñecer los defectos.

Quienes procesan la información se han preocupado siempre de suministrar buenos productos y servicios a sus usuarios. Los resultados del tratamiento documental deben garantizar la calidad y eficacia de los productos que ofrecen si se quiere justificar y asegurar la propia existencia de los repertorios documentales. Entendemos como tales a los documentos referenciales o secundarios que agrupan los registros analíticos de los documentos originales o primarios. Y que lo hacen tanto en el modo más clásico de difusión, sirviéndose de las ediciones impresas (bibliografías analíticas, boletines de resúmenes, sumarios analíticos de publicaciones periódicas), como mediante formatos digitalizados (esos mismos productos fijados en bases de datos referenciales que pueden distribuirse en línea, mediante CD-ROM, e incluso difundirse por Internet). Los repertorios han sido los productos más comunes elaborados en los sistemas de información, y continúan ocupando un lugar fundamental en la difusión de la información científica, técnica y especializada.

1 SWINDELLS, N.- Managing the quality of Information products, en Managing Information, (1995), 4:

${ }^{2}$ UNE-EN ISO 8402: 1995. Gestión de la calidad y aseguramiento de la calidad: Vocabulario. Madrid: AENOR, 1995. 
Para ello debemos decidir la presencia de unos criterios explícitos en las diferentes fases de confección de los repertorios, que a la hora de hacer la evaluación se plasmarán en unos indicadores determinados. La complejidad del proceso es mucha, afectando como elementos a evaluar a:

- La atención, interés, número y pericia de los analistas.

- Las características de las fuentes a incluir en el repertorio.

- Los costes, métodos, procedimientos, y tiempo en que se efectúa el análisis.

- El producto obtenido y su adecuación a los objetivos documentales.

- El esfuerzo que deba hacer el usuario.

- E incluso la forma de presentación.

La calidad de las tareas correspondientes al análisis de contenido documental (especificadas en la indización y el resumen) resulta, pues, fundamental para permitir una satisfactoria recuperación de información y una adecuada explicación de los contenidos a los usuarios. Los rasgos que determinan ambos procesos son paralelos a las exigencias cualitativas que vamos a describir a continuación.

En este sentido, es notable el esfuerzo que actualmente realiza Europa. Sirva de ejemplo la actitud de la European Association of Information Services (EUSIDIC) que se ha convertido en responsable de la excelencia de sus productos y servicios ${ }^{3}$, igual que de la inglesa National Federation of Abstracting and Information Services (NFAIS), así como de laAssociation des documentalistes et bibliothècaires specialisés francesa por asegurar la calidad de los productos generados por sus industrias de la información, en especial por los que conforman las bases de datos.

Nuestra propuesta se centra en la evaluación del proceso de análisis documental, pues sabemos que, dada la complejidad de los procesos de indización y resumen, nunca alcanzan una exhaustividad y precisión plenas. No consideramos aquí otros factores que intervienen en el buen funcionamiento del sistema de información, como

${ }^{3}$ DENIS, S. et al.- Liability in the provision of information services. EUSIDIC Research Project 1989. Brussels: EUSIDIC, 1990. los relacionados con los usuarios. Atendemos tan solo a los factores derivados del análisis documental de contenido.

Precisamente por tratarse de textos, la medición estadística y numérica de los factores cualitativos no siempre puede hacerse. Máxime si consideramos que hablamos de calidad, concepto que conlleva la consideración de rasgos que solo se pueden apreciar mediante la observación y juicio personales. Por ello, la aplicación de estos criterios no tiene por qué ser cuantitativa en exclusiva. A causa de la gran cantidad de información que se acumula en las colecciones documentales, y a la intervención de múltiples factores que hacen de la indización un proceso muy complejo, los procedimientos de recuperación no pueden ser nunca íntegramente exhaustivos y precisos. Así pues, a la hora de determinar el nivel de calidad alcanzado por los productos documentales, debemos considerar unos factores cualitativos que denominaremos criterios, junto a unas unidades de medida o indicadores ${ }^{4}$. Consideramos indicadores de calidad los que miden la coherencia, la pertinencia o precisión, la exhaustividad o respuesta, la consistencia, la densidad informativa, la profundidad, la extensión o tamaño, así como los indicadores temporal, de costes (recursos invertidos en un servicio), del esfuerzo del usuario y de errores.

El indicador temporal es la medición del tiempo de respuesta que está determinado por la organización y el tipo de archivo en que se custodien los datos, la ubicación del sistema de información, la saturación a que el servicio se halle expuesto y el tamaño de los ficheros manejados.

Mientras que el esfuerzo del usuario vendrá determinado por la ayuda que le puedan prestar los profesionales de la información, la cantidad de información que se le sirva tras efectuar una búsqueda, el formato en que la información se presente, así como la facilidad de manejo e interacción con el sistema, y las propias habilidades para buscar en general o para hacerlo en un sistema en concreto.

El indicador de errores quiere valorar la introducción de errores que modifiquen los rasgos originales de la información analizada. Suceden en cada eslabón de la cadena documental: en la identificación de las fuentes, en los procesos de extracción, clasificación y codificación, e incluso en la grabación de los datos.

\footnotetext{
${ }^{4}$ Un buen indicador debe ser pertinente hacia su objeto, operativo y cuantificable.
} 


\section{PROPUESTA METODOLOGICA}

A. Tareas de identificación de los repertorios existentes en el área o las áreas estudiadas (incluyéndose las Bibliografías y las Bases de Datos - ya sean estas on line, en CD-ROM, o distribuidas por Internet-). Confección del listado de los repertorios identificados.

\section{La identificación pretende obtener conocimiento sobre estos aspectos del repertorio:}

\section{A.1. Antecedentes del mismo. Su evolución.}

A.2. Naturaleza de la Bibliografía o Base de Datos:

- ¿Qué campo cubre?

- ¿Quiénes son los editores y quiénes los usuarios potenciales?

- ¿Qué tipo de producto concreto nos ofrece?

- Contenidos.

- Formato.

- Acceso.

- Periodicidad.

- ¿Cómo podemos obtener la información de esos productos?

A.3. Medición del porcentaje de exhaustividad de la cobertura del repertorio (CR): es el grado de cobertura temática (Coverage) o proporción de información existente sobre una materia, publicada dentro de un período de tiempo concreto, que está incluida en la Base de Datos. Se realiza mediante la construcción de una lista de publicaciones periódicas del área estudiada, comparándola con las que recoge el repertorio. De acuerdo con la fórmula:

$$
\mathrm{CR}=\mathrm{C} / \mathrm{i} \times 100
$$

donde $\mathbf{C}$ es el número de publicaciones periódicas del área recogidas en el repertorio e $\mathbf{i}$ el número total de publicaciones periódicas del área. Esta unidad de medida tiene una apreciación cualitativa, al tener que considerarse si las publicaciones periódicas recogidas son después analizadas en su integridad o lo son de manera selectiva.
Cuando se hagan estudios comparativos de cobertura entre varios repertorios estaremos intentando delimitar la cobertura que efectúa cada uno de ellos y, en consecuencia, también las repeticiones y lagunas contenidas en los listados de referencias respecto de los originales de las áreas que pretenden cubrir. En este caso hablaremos de cobertura relativa, en la que dados dos repertorios A y B, la cobertura de A será :

Número de referencias en $\mathbf{A}$

\section{Número de referencias en A UB}

Asimismo, podremos utilizar la medida complementaria del solapamiento, por la que se perciben aquellas referencias comunes a dos o más sistemas de información. Dados dos repertorios A y B, el solapamiento global saldrá del cociente:

Número de referencias comunes en A y B

Total de referencias en A U B

Mientras que el solapamiento relativo será el de un repertorio $(\mathrm{A})$ respecto a su propia cobertura ${ }^{6}$ :

$$
\text { Número de referencias comunes en A y B }
$$

\section{Total de referencias en A}

Contraria al solapamiento global es la medida del aporte específico de un repertorio (A), resultante de oponer

Número de referencias no solapadas comunes en A

$$
\text { Total de referencias en } \mathbf{A} \mathbf{U} \mathbf{B}
$$

Una consecuencia del estudio de las revistas de un área mediante la cobertura y el solapamiento en los repertorios de esa misma área es saber cuáles son las revistas más analizadas y, por tanto, las que forman el núcleo dentro de una disciplina.

B. Preparación profesional de los técnicos que elaboran el repertorio. Debe entenderse que el primer criterio de calidad se deriva de la actitud y preparación específicas con que los analistas abordan su trabajo:

${ }^{5}$ ABAD GARCÍA, F.- Investigación evaluativa en Documentación. Valencia: Universitat de Valencia, 1997: 130-132.

${ }^{6}$ LABOIRE, T.; HALPEIN, M. y WHITE, H.- Library and Information Science Abstracting and Indexing services: Coverage, Overlap and Context, en Library and Information Science Abstracts, (1985), 7: 183-195. 
- Si el repertorio está realizado por profesionales o no.

- Si son graduados en Biblioteconomía y Documentación.

- Si lo son en el área de la Base de Datos o Repertorio.

- Si no tienen titulación universitaria, pero han sido formados en la editorial.

- Cuál es su nivel de experiencia, conocimiento de la terminología y de las reglas de uso del lenguaje utilizado.

- Cuál es la relación entre el número de analistas y el trabajo a realizar.

- Grado de atención e interés.

Obtener información de este tipo es una tarea difícil que muchas veces conllevará la interrogación mediante encuesta a los propios servicios de información, ya que nada suele explicitarse de este y otros asuntos en las explicaciones técnicas que ofrecen los repertorios.

C. Existencia de normativas o directrices: observar si se guarda respeto a las normas generales y a las directrices existentes en el centro productor; cuál es la estabilidad de los criterios fijados en ellas; qué seguimiento o supervisión de su cumplimiento se realiza; si existe o no algún control periódico de la calidad ${ }^{7}$. Todos ellos son factores que definen la homogeneidad en los procesos de indización y resumen, así como en la publicación de los resultados.

D. Tipo de lenguaje utilizado para efectuar la indización:

- Mediante lenguaje libre:

- Extraído de las palabras del título.

- A partir de la información contenida en el resumen.

- Considerando el texto completo.

Se pretende saber de qué manera se indizó en lenguaje libre. En este caso, se deben contrastar las palabras-clave con el título, el resumen o el propio texto para determinar si se deben a un proceso de extracción, a una determinación de los documentalistas, o bien si son las mismas palabras-clave utilizadas por el autor del original.

- O usando algún lenguaje controlado, cuya identificación y características deben determinarse, y que puede ser:

${ }^{7}$ LANCASTER, F. W.- El control de vocabulario en la recuperación de la información. Valencia: Universitat de Valencia, 1996.
FIGURA 1

Comparación de las características fundamentales entre los tipos básicos de resumen.

\begin{tabular}{lll}
\hline & Estructuras & Expresión \\
\hline Informativo & $\mathrm{Mg}+(\mathrm{Mp} 1+\mathrm{Mp} 2 \ldots+\mathrm{Mpn})=$ & \\
Explicativa & $\mathrm{Mg}+5$ & \\
Selectivo & $\mathrm{Mg}+(\mathrm{Mp} 1+\mathrm{Mp} 2 \ldots+\mathrm{Mpn})=$ & Indicativa \\
& $\mathrm{Mg}+\mathrm{Sp}$ & Indicativa \\
Indicativo & $\mathrm{Mg}(+$ otra información $)$ & \\
\hline
\end{tabular}

- Tesauro.

- Lista de palabras clave.

- Glosario de descriptores.

- Lista de encabezamientos de materia.

- Otros (Mapas conceptuales, CDU, ...).

La accesibilidad al lenguaje documental vendrá determinada por los recursos utilizables por el usuario para la selección de los términos de recuperación más adecuados.

E) Tareas relativas a la evaluación del análisis de contenido (indización y resumen, cuando haya). Dado que se trata de una apreciación cualitativa, se requiere la selección de un número suficientemente representativo de registros de cada Base de Datos o Bibliografía atendida, y su posterior comparación con los artículos originales a los que esos registros se refieren. La evaluación se hace mediante inspección directa de la muestra de los registros seleccionados, que es considerada representativa de cada repertorio. La aplicación de este método muestra mucha lentitud, pero nos asegura que el proceso sea crítico y deductivo.

\section{CRITERIOS A EVALUAR EN LA ELABORACIÓN DE LOS RESÚMENES}

1) Debe considerarse el grado de reutilización de los resúmenes hechos en origen (de autor). Hay que apreciar si se aprovechan tal cuál estaban en el original, o si por el contrario se han mejorado técnicamente, buscando las deseables coherencia y normalización ${ }^{8}$. El seguimiento

8 MOREIRO, J.; MELO, D.; GARCÍA, J.; DUARTE, E.; ALBUQUERQUE, E.; MELO, L. e NEVES, D.- Avaliação dos repertórios brasileiros em Agricultura, Ciência da informação e Direito: A qualidade da análise de conteúdo, en Ciência da Informação, (1998), 4: 44. 
de la reutilización de los resúmenes debe discernir entre aquellos copiados literalmente del autor, de aquellos que basándose en el resumen analítico han sido corregidos o modificados. En el caso de que el original no contenga resumen de autor, sería muy positivo comparar el resumen de la referencia con los primeros párrafos del texto o alguna frase significativa en la que se represente la información sustancial. Ya que muchas veces es esta la fuente de la que se extraen los datos que luego conforman un resumen, casi siempre indicativo.

2) Es fundamental identificar cómo se ha efectuado el traslado de la superestructura del original (de manera informativa, selectiva o indicativa $)^{9}$ :

- Los resúmenes informativos deben trasladar la Macroestructura global del texto $(\mathrm{Mg})$, además de la superestructura $(S p)$, o lo que quiere decir además de las macroestructuras parciales $(\mathrm{M} p)$ ordenadas, y tener una expresión explicativa (con oraciones suficientemente informativas). Es en realidad una versión abreviada de la idea central de un documento (macroestructura global), así como de las ideas que vinculan cada una de las partes que lo componen (macroestructuras parciales), y éstas en orden (superestructura). Así pues, el resumen informativo representa: Macroestructura global + (Macroestructura parcial $1+$ Macroestructura parcial $2 \ldots+$ Macroestructura parcial $n$ ), o lo que es lo mismo Macroestructura global + Superestructura, junto a una descripción concisa. Esquemáticamente, y en el caso de un artículo de ciencia experimental, las oraciones del resumen informativo indican:

\section{Macroestructura global / objetivos}

2. Metodología: Fase de descripción / Fase de análisis.

3. Resultados - Discusión.

4. Recomendaciones - Conclusiones.

5.Bibliografía - Anexos.

- Los resúmenes selectivos deben trasladar la Macroestructura global del texto $(\mathrm{Mg})$, además de la superestructura $(S p)$, o lo que quiere decir además de las macroestructuras parciales $(\mathrm{Mp})$ ordenadas), pero hacerlo en expresión indicativa. Resumen selectivo $=\mathrm{Mg}+$ $(\mathrm{Mp} 1+\mathrm{Mp} 2 \ldots+\mathrm{Mpn})$, o lo que es lo mismo $\mathrm{Mg}+\mathrm{Sp}$, con explicaciones para esta última tan sólo indicativas.

${ }^{9}$ MOREIRO GONZÁlEZ, J. A.- Aplicación de las Ciencias del texto al resumen documental. Madrid: Universidad Carlos III - Boletín Oficial del Estado.
- Los resúmenes indicativos se limitan a trasladar la Macroestructura global del texto $(\mathrm{Mg})$ y, a veces alguna de las macroestructuras parciales $(\mathrm{Mp})$, siempre mediante una expresión indicativa $(\mathrm{Mg}+$ otra información, explicada ésta solo de forma indicativa).

3) Calidad técnica de los resúmenes: Viene dada por aquellas presencias inútiles y ausencias evitables, antes explicadas ${ }^{10}$ : presencia de redundancias (frases inútiles, repetición del título, presencia de expresiones como: El autor analiza ..., Este artículo ..., El documento que ...); presencia de faltas ortográficas o sintácticas. Debe hacerse explícito en la frase introductoria del resumen la naturaleza y enfoque del documento original ${ }^{11}$. Así, debe comenzarse el resumen indicando la naturaleza del documento original: si se trata de un artículo, crítica histórica, crónica, entrevista, editorial, ensayo, estudio estético, examen de un caso, nota, presentación de resultados, reportaje, tesis,... Luego, debe constatarse el enfoque específico que caracteriza al trabajo analizado, cómo el autor ha tratado el asunto: desarrollo complementario, estadístico, en exhaustividad, expositivo, de réplica, revisión, técnico-experimental, teórico, ...

4) Tamaño de los resúmenes: cantidad de palabras que los conforman, pareciendo excelente que su extensión se sitúe entre las 100 y las 250 palabras ${ }^{12}$. Considerando el número total de palabras que conforman un resumen, la densidad es el indicador que mide las que son nocionales, es decir, aquellos términos que representan un concepto relacionado con el tema o materia de la que se trata. La fórmula de la densidad es:

Número de palabras nocionales

$\frac{\text { Número total de palabras }}{2} 100$

5) Indicadores de legibilidad de los resúmenes. La legibilidad mide la claridad de expresión, y en el caso de los resúmenes es un requisito de su calidad expresiva, por lo que asimismo puede afectar a la indización. Consideramos que actúan como factores indicativos de legibilidad $^{13}$ :

${ }^{10}$ MOREIRO GONZÁlEZ, J. A.-La técnica del resumen científico, en LÓPEZ YEPES, J.- Manual de Información y Documentación. Madrid: Pirámide, 1996: 383.

${ }^{11}$ MOREIRO GONZÁleZ, J. A.- Aplicación de las Ciencias del texto al resumen documental. Madrid: Universidad Carlos III - Boletín Oficial del Estado.

${ }^{12}$ TENOPIR, C. y JACSÓ, P.- Quality of abstracts, en Online Review,(1993), 5: 46.

${ }^{13}$ Ibid.Id.: 50. 
Dificultándola

Facilitándola

- La presencia de la voz pasiva

- La subordinación excesivade oraciones dentro de una frase.

- La presencia de oraciones cortas.

- La presencia de abreviaturas

y acrónimos sin normalizar.

La legibilidad debe alcanzar a la medición del número de sílabas por palabra, siguiéndose por el número de palabras por frase, y concluyendo por el número de frases por párrafo. Si bien creo que debe centrarse exclusivamente en el recuento del número de palabras por frase, de acuerdo con estas consideraciones:

Tendremos por oraciones cortas las inferiores a 15 palabras.

Por oraciones medias, las que tengan entre 15 y 20 palabras.

Y por oraciones largas, las que tengan más de 30 palabras

(El porcentaje se obtendrá por repertorio)

6) La cohesión de los resúmenes consiste en comprobar que las oraciones estén bien unidas gramaticalmente Es un aspecto puramente de coherencia superficial que permite la lectura seguida, de manera discursiva, sin trabas y que se considera, por tanto, dentro de los valores expresivos de la legibilidad.

\section{CRITERIOS E INDICADORES CON LOS QUE SE HIZO LA INDIZACIÓN}

El proceso de indizar consiste en describir y caracterizar un documento con la ayuda de representaciones de los conceptos contenidos en dicho documento, con la finalidad de permitir una búsqueda eficaz de las informaciones contenidas en una colección documental. Debe destacarse aquí la relevancia que alcanza una indización correcta a la hora de evaluar un repertorio. Ya que la indización se establece como instrumento referencial del conocimiento de los contenidos específicos de cada repertorio. Razón por la que cumple el papel protagonista en la recuperación de los contenidos.

El indizador debe buscar y utilizar un descripción que traduzca lo más de cerca posible el contenido del documento (especificidad), rechazando los descriptores demasiado generales o demasiado particulares con relación a las nociones que expresa el documento. Su logro supone la relevancia, concepto que puede atribuirse a la recuperación, cuando un documento es útil para los propósitos que causaron una búsqueda por parte del usuario $^{14}$. Lo ideal sería encontrar todos los documentos relevantes y evitar los no relevantes (obtener a la ver exhaustividad y precisión). Pero que en nuestro caso lo aplicamos a la carga de significación del descriptor.

1) La procedencia de los términos de indización es un elemento que marca diferencias para valorar la calidad de un repertorio. Lo es por la enorme importancia de una recuperación exacta, sin la cual las demás tareas en la construcción del repertorio no tienen sentido. Respecto a este punto puede darse varias situaciones:

- Que los indizadores reutilicen la indización hecha en origen. En cuyo caso habrá que ver si fue corregida o no. Y dependerá también de cómo se efectuó esta indización en origen: si lo fue con descriptores o con palabras clave. En este último caso sería adecuado revisar desde el original la pertinencia y exactitud de las palabras escogidas para representar los conceptos. Una indización en palabras clave que traslade literalmente la propuesta por el original puede dejar pasar incoherencias e imprecisiones.

- Si percibimos que la indización fue totalmente elaborada en el centro de análisis, habrá que plantearse la procedencia de los descriptores, por una parte, respecto al lenguaje documental utilizado (comentado antes, y que puede darse una presencia simultánea de descriptores y palabras clave en un mismo registro) y, por otra, intentar determinar si en cualquiera de los dos casos fueron obtenidos a partir de la consulta al texto íntegro, desde los epígrafes del original, consultando tan solo la introducción o el resumen, o si exclusivamente se tomó el título como fuente de consulta. Estas últimas consideraciones guardan una relación muy estrecha con el punto siguiente.

2) Respecto a la profundidad en la representación del contenido textual (hasta qué nivel se representa la superestructura), dependerá de si los términos de indización se refieren a todo el texto reflejando las macroestructuras parciales, si fueron extraídos a partir solamente del resumen analítico y, en dependencia del tipo de este, que alcancen a representar solo algunas de las macroestructuras parciales, o si son tan genéricos que reflejen tan solo los conceptos del título.

\footnotetext{
${ }^{14}$ PÉREZ ÁLVAREZ.OSORIO, J.- Introducción a la información y Documentación científica. Madrid: Alhambra, 1988.
} 
El indicador de profundidad resulta del cociente:

Número de palabras del original

$$
\text { Número de palabras x } 10
$$

Esta cualidad se asocia directamente con la pertinencia o relación entre los contenidos del original y los términos de la indización ${ }^{15}$.

3) Índice de consistencia, que solo sería posible en aquellas áreas con varios repertorios, la consistencia del análisis documental se refiere a que un concepto o tema aparece siempre expresado de la misma forma ${ }^{16}$ :

$$
\text { IC (\%): } \frac{100 A}{A+M+N}
$$

Donde A son los términos comunes a los repertorios $\mathrm{M}$ y $\mathrm{N}$.

En el caso de que la descripción fuese comparativa entre varios indizadores o usuarios estaríamos hablando de la uniformidad u homogeneidad con la que todos ellos deben describir el mismo documento, o documentos sobre el mismo tema, de la misma manera.

\section{4) Otros indicadores y factores de evaluación.}

\section{a) Indicador de pertinencia o precisión.}

Tras obtener la respuesta a una búsqueda, es un cociente (precision ratio) que resulta de dividir el:

Número de documentos relevantes recuperados

Número total de documentos recuperados

Desde la perspectiva opuesta a la pertinencia, puede hablarse también de una tasa de silencio: los documentos no recuperados, pero que son relevantes. El cociente de precisión nos indica que cuanto más se acerque a 1 mejor será la recuperación.

85 referencias pertinentes

ejemplo: $-=0,6-0,7$ (resultado bueno) 100 referencias totales

${ }^{15}$ LANCASTER, F. W. - Indización y resúmenes: teoría y práctica. Buenos Aires, EB publicaciones, 1996: 54.

${ }^{16}$ ABAD GARCÍA, F.- Investigación evaluativa en Documentación. Valencia: Universitat de Valencia, 1997: 130.
Es un concepto relativo que se mide con respecto a un referente (serie de datos) que sirve como punto de partida. Se puede hablar de pertinencia de un descriptor, de la indización, del lenguaje, ...

\section{b) Indicador de exhaustividad o de respuesta.}

El indicador de exhaustividad (recall ratio) busca que todos los temas, objetos y conceptos que encierra el documento estén bien determinados en la indización, por lo que habrá una respuesta ajustada a una búsqueda dada, que se mide en porcentaje a través de la relación entre:

Número de documentos relevantes recuperados

Número total de documentos relevantes existentes en la Base de Datos

Es más difícil de corregir que en el caso de la pertinencia, pues para comprobarlo habrá que examinar toda la colección documental. También un porcentaje de respuesta entre el 0,6\% y el 0,7\% se considera un buen resultado. Respecto a este indicador, y desde la perspectiva opuesta a la exhaustividad, hablaremos de una tasa de ruido que valora la proporción de documentos recuperados que no son relevantes para la búsqueda propuesta:

Número de documentos no-relevantes recuperados

Número de documentos recuperados

Se ha demostrado que la eficacia es mayor utilizando lenguajes de términos simples que cuando se manejan lenguajes de términos sintagmáticos ${ }^{17}$. Así como se ha observado la existencia de una relación inversa entre la precisión y la exhaustividad.

c) Finalmente, podemos hablar del esfuerzo requerido en la recuperación (Retrievability): cantidad de información contenida en la Base de Datos, sobre un tema concreto, que puede recuperarse usando una estrategia de búsqueda razonable.

Así como de la facilidad para juzgar la pertinencia (Predictability): recursos utilizables por el usuario para juzgar con rapidez si los registros son adecuados a sus necesidades.

Y de la actualización (Timeliness): presencia y proporción de la información más moderna dentro del conjunto de registros consultables en la Base de Datos.

${ }^{17}$ ELLIS, D.- New horizons in information retrieval. London: Library Association, 1991. 


\section{PRESENTACIÓN COMPARATIVA DE LOS RESULTADOS OBTENIDOS}

Una parte de las conclusiones ha de expresarse en textos exponiendo valorativamente los resultados tras hacer observaciones en los repertorios siguiendo los criterios antes expresados.

Deben distinguirse los resultados de la aplicación de los criterios a los juicios de calidad del repertorio entendido globalmente de aquellos propios del resumen o de los que afectan a la indización. Es conveniente separar la aplicación de los criterios en la observación de un hecho o descripción técnica de las mediciones en que se aplicaron índices o indicadores.

Es aconsejable utilizar tablas para recoger los resultados de la evaluación de cada una de los repertorios o incluso de cada uno de los registros analizados. Como ejemplo:

\begin{tabular}{llll}
\hline & BD o registro 1 & BD o registro 2 & Etc \\
\hline Reutilización & $\mathrm{Si}$ & No \\
Traslado de ba superestructura & $\mathrm{Si}$ & $\mathrm{Si}$ \\
Tipo de resumen & Indicativo & Selectivo \\
Cohesión & Aceptable & Escasa \\
Número de palabtas & 105 & 98 \\
Legibilidad & Buena & Buena \\
Exhaustividad & 0,5 & 0,8 \\
Precision & 0,6 & 0,7 \\
Etc & - & \\
\hline
\end{tabular}

La utilización de gráficas comparativas y de tortas por porcentajes otorga much'a claridad a las conclusiones y facilita la comprensión de los resultados por parte de los lectores.

Artigo aceito para publicação em 06-02-2001

\section{REFERENCIAS BIBLIOGRÁFICAS}

ABAD GARCÍA, F. Investigación evaluativa en documentación. Valencia : Universitat de Valencia, 1997. p. 130-132.

AENOR (Madrid). UNE 50-121-91: métodos para el análisis de documentos, determinación de sua contenido y selección de los términos de indización. Madrid, 1991.

ISO 8402:1995: gestión de la calidad y aseguramiento de la calidad - vocabulario. Madrid, 1995.

AGUADO BLANCO, Maria B. Técnicas avanzadas de indización y clasificación de información. Madrid : Aguado, 1997.

BAKER, S. The measurement and evaluation of library services. Arlington : Information Resources, 1993.
BLAIR, D. Language and representation in information retrieval. Amsterdam : Elsevier, 1990.

CHAUMIER, J. Análisis y lenguajes documentales. Barcelona : Mitre, 1986.

DENIS, S. et al. Liability in the provision of information services. Brussels : EUSIDIC, 1990.

(EUSIDIC Research Project, 1989).

ELLIS, D. The effectiveness of information retrieval systems: the need for improved explanatory frameworks. Social Science Information Studies, n. 4, p. 261-272, 1984.

New horizons in information retrieval. London : Library Association, 1991

EISENBERG, M. Measuring relevance judgments. Information Processing and Management, n. 24, p. 373-389, 1988.

FOLSTER, M. B. A study of the use of information sources by social science researchers, Journal of the Academic Librarianship, n. 1, p. 7 11, 1989.

GRIFFITHS, J.; KING, D. A manual on the evaluation of information centers and services. Neuilly-sur-Seine : North Atlantic Treaty Organization, 1991.

INFORMATION MARKET OBSERVATORY. The quality of electronic Information products and services. Luxembourg, 1995. (Working Paper, 95/4).

LABOIRE, T.; HALPEIN, M.; WHITE, H. Library and information science abstracting and indexing services: coverage, overlap and context. Library and Information Science Abstracts, n. 7, p. 183-195, 1985.

LANCASTER, F. W. El control de vocabulario en la recuperación de la información. Valencia : Universitat de Valencia, 1996.

Indización y resúmenes: teoría y práctica. Buenos Aires : EB Publicaciones, 1996.

MICHEL, J. ; SUTTER, E. Valeur et compétitivité de l'information documentaire. Paris : ADBS, 1991.

MOLINA, M. Indicadores de calidad descriptiva en la gestión de los procesos analítico-documentales. In: ACTAS DE LAS JORNADAS ESPAÑOLAS DE DOCUMENTACIÓN, 1994, Gijón. Oviedo: Universidad, 1994. p. 184-204.

MOREIRO GONZÁlEZ, J. A. Aplicación de las ciencias del texto al resumen documental. Madrid : Universidad Carlos III, [1996?]. Boletín Oficial del Estado.

La técnica del resumen científico. In: LÓPEZ YEPES, J. Manual de información y documentación. Madrid : Pirámide, 1996. p. 373-390.

et al. Avaliação dos repertórios brasileiros em agricultura, ciência da informação e direito: a qualidade da análise de conteúdo. Ciência da Informação, Brasília, v. 27, n. 3, p. 284-292, set./dez. 1998.

O'CONNOR, B. Explorations in indexing and abstracting: pointing, virtue and power. Englewood: Libraries, 1996.

PÉREZ ÁLVAREZ, J. Osorio. Introducción a la informacióon y documentaciíon científica. Madrid : Alhambra, 1988.

ROLLING, L. Indexing consistency, quality and efficiency. Information Processing and Management, v. 17, p. 69-76, 1981.

SUTTER, E. Maîtriser l'information pour garantir la qualité. Paris : AFNOR, 1992.

SWINDELLS, N. Managing the quality of information products. Managing Information, n. 4, p. 36-48, 1995.

TENOPIR, C.; JACSÓ, P. Quality of abstracts. Online Review, n. 5, p. 44-55, 1993.

UNESCO (Paris). Principes directeurs pour l'évaluation des systèmes et services d'information. Paris : UNESCO, 1978. 